\title{
Vaccination Policy for Smallpox
}

FEAR of smallpox has again renewed controversy on the policy of routine vaccination against this infection in nonendemic areas such as the United Kingdom. Smallpox ceased to be endemic in this country in 1935 and all but two outbreaks, including the recent outbreak which presumably originated in a laboratory at the London School of Hygiene and Tropical Medicine, have arisen from importations. Indeed, since 1950 there have been 378 cases of smallpox in the United Kingdom resulting in 60 deaths. Alarming as these figures appear, the risks of vaccination complications may nonetheless outweigh the benefits of routine vaccination in non-endemic situations. Thus between 1951 and 1970 there were about 100 deaths from smallpox vaccination in England and Wales (Dick, Brit. med. J., 3, 163 ; 1971). Even so, these figures may be incomplete because there is probably some under-reporting, and non-fatal complications have been excluded. This is still perhaps not the best way to balance the policy of universal vaccination, the risk of smallpox and the complications of the vaccination procedure itself.

Herd immunity is still a common philosophy, and there is little doubt that a vaccinated community has an advantage, as far as mortality rate is concerned, over an unvaccinated community. It has been estimated, however, that in 1947, even after allowing for mass vaccination during the war and endemic variola minor during the period 1920 to 1934, the total immunity in England and Wales was less than 20\% (Dixon, Smallpox, Churchill, London, 1962, p. 342). The acceptance rate for primary infant vaccination has been perhaps $40 \%$ and for revaccination probably less than $10 \%$. In order to establish a high herd immunity it would be essential to have successful universal revaccination, every 3 years, throughout life. But even apart from the risks of vaccination it is difficult to justify immunization against a disease which has been successfully eradicated from all but seven countries-Bangladesh, Botswana, Ethiopia, India, Nepal, Pakistan and Sudan (World Health, October 1972). Because of the success of the eradication programme of the World Health Organization and the concomitant decline in the risk of smallpox importation, the United States and the United Kingdom discontinued in 1971 the practice of routine vaccination.

Severe outbreaks of smallpox from importations have been controlled for many years now by isolating cases, and tracing, vaccination and surveillance of known probable contacts. This policy of ring vaccination has been remarkably effective. There is, therefore, no place for mass unselective vaccination during a limited outbreak. Following the recent London incident some informed newspapers attempted to discourage mass vaccination, unlike the situation accompanying the outbreak of smallpox in South Wales in 1962, when at least eighteen people died from indiscriminate vaccination (Dick, loc cit.), generated to a large extent by panic.

At the same time it is imperative to vaccinate and revaccinate and to ensure successful vaccination of high- risk groups such as doctors, nurses, laboratory workers, ambulance drivers and other hospital and public health personnel. In Europe during the period 1950 to 1971 , for example, smallpox killed, among the hospital staff, an alarmingly high proportion of its young adult victims, fourteen or $70 \%$ of the twenty without previous vaccination (Mack, J. infect. Dis., 125, $161 ; 1972$ ). The policy of selective and ring vaccination must continue, therefore, at least for the time being.

\section{From our Medical Virology Correspondent}

\section{Diamond and Cavitation}

WHY are diamonds found naturally in geological circumstances which belie the high pressures needed to synthesize them? This is the question which Galimov considers on page 389 of this issue of Nature. He comes to the interesting conclusion that diamonds could form when cavitation bubbles in flowing kimberlite magma collapse. The point is that cavitation can occur when the pressure on a liquid with gas dissolved in it is reduced below some critical value. Cavitation can take place, for example, in the vicinity of propeller blades on ships and in those circum. stances can cause considerable damage.

Kimberlite usually occurs in vertical pipes a few tens of metres in diameter and a few kilometres long. Galimov calculates that magma flowing at a pressure of $20 \mathrm{kbar}$ or so could be induced to cavitate if the magma passed through a constriction the cross-section of which is only two to three times smaller than that of the neighbouring regions of pipe. If one applies the celebrated Bernoulli relation it turns out that the pressure in these circumstances is reduced below that required for complete dissolution of carbon dioxide at $1,000^{\circ} \mathrm{C}$. Further calculations reveal that the pressures and temperatures reached locally when cavitation bubbles collapse would be such as to allow the synthesis of diamonds.

Galimov also deals with the question whether the extreme conditions that exist when a cavitation bubble collapses last for long enough for a diamond to form. Extrapolating from the conditions in which diamonds have been artificially created in explosions, he calculates that appropriate natural conditions probably exist for a few milliseconds at a time, three orders of magnitude longer than is the case in a typical explosion experiment.

The cavitation concept also explains why some diamonds seem to have grown in a series of discrete steps: a newly formed diamond would evidently be a good site for the formation of subsequent cavitation bubbles and during each collapse the diamond would increase in size. And because the high pressures only occur very locally, other minerals usually formed in such conditions are not found in kimberlite. 